\title{
CONTEMPORARY TRENDS OF VLOGGING IN PAKISTAN: A CONTENT ANALYSIS OF POPULAR VLOGS
}

Received: 22-Dec-2020 | Accepted: 05-Jan-2021

Kanwal Ilyas $^{1}$ | Dr. Asmat Ara ${ }^{2}$

\begin{abstract}
This research is aimed to study the contemporary trends of VLogging in Pakistan in light of audience engagement. Using the quantitative content analysis approach, the themes of the most popular VLog channels and video blogs were to be determined. The sample is selected with Purposive Sampling Techniques, which allowed the researchers to study the fifty most popular VLogs produced by Pakistani VLoggers. This research study proved to be significant in understanding the ongoing impact of VLogs as a medium in Pakistan and its future scope in the country's multifaceted media industry. With such results, it is to be determined whether VLogs can be used to effectively disseminate the information and, if they can, in what direction they are creating the most impact.
\end{abstract}

Keywords: VLogging, YouTube, Subscription, VLogging Trends, VLoggers, Pakistan, VLog style

\footnotetext{
Author's Affiliation:

Institution: University of Karachi ${ }^{1,2}$,

Country: Pakistan ${ }^{1,2}$

Corrsponding Author's Email: *
}

The material presented by the author(s) does not necessarily portray the view point of the editors and the management of the ILMA University, Pakistan.

2709-2232 (Online) (C2021, published by the ILMA University, Pakistan.

This is open access article under the license. https://creativecommons.org/licenses/by/4.0/ 


\section{INTRODUCTION}

VLogging has become one of the emerging and popular unconventional methods of conveying direct messages to the audience. A VLog shows an individual in front of the camera, typically talking and sharing about different topics, either related to the individual himself or the world. (VLog Brothers, 2008). Video blogs or VLogs are the direct results of modern age innovation and personal creativities (Stanley, 2016). A blog is a text accompanied by images or graphics, written in a conversational or informal style (Dave,2003). It gave every writer space outside the set boundaries to reach out to the public on various issues, not being shared or highlighted on conventional media.

By definition, a VLog is a video or a set of videos that are regularly posted on the internet. Such videos are recorded on personal thoughts, experiences, or any issue in particular. (Medoff, et al., 2007). Vlogs are described as the video collections that serve as an audiovisual life documentary and a vehicle for communication and interaction for the users on the Internet (Biel and Perez, 2010). VLogs are also described as the "video version of text-based weblogs" that appears on the first examination as no different from existing media (Moor et al., .2010). VLogs have been termed as the 'conversational video blogs' (Biel \& Perez, 2010) and even as monologues like videos (Aran et al., 2014). They are defined in the Cambridge dictionary as a "record of the thoughts, opinions, or experiences that are filmed and published on the internet (Cambridge English Dictionary, 2016). VLog is an acronym for video blogs, which are short online videos created to educate, review, advise, market, or entertain people (Stanley, 2016). According to Michael Sean Kaminsky (2010), people blogs through videos to express themselves, entertain, connect with people, make money, teach, promote a specific cause, or sometimes just for fun.

The history of VLogs is not as old as other media. The first-ever video blog shared on the web was posted on January 2, 2000, by Adam Kontras (Maslanka, 2017). He recorded his cross-country travel while moving to Los Angeles in his pursuit of show business. However, not until 2005, VLogging touched the heights of popularity. It was all due to the initial launch of YouTube - A video posting and sharing engine (Stanley, 2016). Youtube has provided the largest space for all the web users to directly the cyber audience. Later the website officially acknowledged the creative VLoggers and made the site open for business.

In Pakistan, VLogging emerged as a success back in late 2016 or in the early days of 2017, when video sharing platforms like Youtube offered monetization services to the content producers. VLoggers gained such a wide scale of popularity over the years that some of them came close to television or movie stars or celebrities. This research paper is designed to discover all the popular niche of VLogs in Pakistan.

\section{VLoggers:}

According to the above-given definition of VLogs, a VLogger can be any person creating, producing, directing, recording, and uploading a VLog or video blog on the internet. From a research perspective, this definition is not appropriate to 
quantify all types of VLoggers. To avoid any possible anomalies in the results and to exterminate contaminating variables, only VLoggers belonging to Pakistan and living in the country with a non-celebrity status are studied under the ambit of this research.

\section{VLog and YouTube Channels:}

YouTube is an American video-sharing website that was created back in 2005. It has its headquarter stationed in San Bruno, California. Created on the 14th February 2005, by three former PayPal employees, including Steve Chen, Chad Hurley, and Jawed Karim, it was later merged with google, which further expanded. Like conventional television channels, YouTube also provides a separate space to each of its content producers exclusively to upload their work without any external influences. Each channel is associated with a name assigned by the creator himself. The pace of running the channel is also dependent on the mindset and efforts of the content producer. When initiated by a VLogger, such a channel can be termed as a 'VLog channel.' A single channel can contain millions of videos, which can all be VLogs, or it can only contain a single section comprising of VLogs.

\section{YouTube Subscription:}

Subscription is an agreement to either receive or be given access to information or services for a specific period, especially over the internet (Oxford English Dictionary, 2018). When this definition is applied to the analysis of YouTube videos, the subscription can be defined as getting direct access to the services and information upload on the YouTube channel through notifications by clicking the subscription option. Any YouTube channel subscribed by the greatest number of people would indicate the greater interest of the audience in the content produced by that channel.

\section{Scope and Significance of the Research}

Ever since the monetization services have been offered in Pakistan by Youtube, there has been a great influx of video bloggers from different parts of the country who produce online video content using different themes and styles. As video blogging emerged in Pakistan in the recent past, its effectiveness as a medium is still not known to many. Besides the efficacy of this medium, the various trends and themes covered are also in the dark.

To understand the future success and implications of Vlogging in the country, it is important to analyze the content produced by the Pakistani Vloggers. Therefore research is carried out to assess the type of content produced through vlogging and analyze the connection between certain themes and styles of content production with audience engagement. And that will help us visualize the future scope of vlogging in Pakistan and the extent to which Vloggers as a content producing community can best engage the audience.

The results from this research will also help future vloggers to produce their content in the light of the prevailing trends while keeping in view the audience's engagement, likes, or dislikes. It will also help the existing vloggers improve their content to provide quality services to their audience. This study will also serve as 
preliminary research on vlogging in Pakistan, which can then be expanded and used to analyze the future vlogging trends and draw a comparison between the trends in Pakistan and those prevalent worldwide.

\section{LITERATURE REVIEW}

Maggie Griffith, and Zizi Papacharissi (2010), examined the culture and trend of video blogging while analyzing the language, communication style, and rhythm of the video blogs. The researchers had selected ten personal VLogs and deconstructed them into links, text, site, videos, and comments. As a result of this analysis, the researchers discovered three prominent themes, including VLogs as diaries, a medium of identity expression, and a tool to indulge in narcissism. They randomly selected ten VLogs from different sources. The statistics proved that VLog is a complex medium of self-expression. Despite its complexity, individuals seek this new medium more often due to its enhanced interactivity with the audience and wide space available for all forms of self-expression.

Qiang Yang and his colleagues (2010) carried out a qualitative study on VLogs as a new technological innovation in the media industry. The study specifically targets the four key elements which posses' different challenges to VLogging. After analyzing the obstacles, researchers tactfully offered various solutions to the stated problems. They advised better interactivity, scalability, accessibility, and searchability.

Heather Molyneaux, Susan O’Donnell, Kerri Gibson, and Janice Singer (2016), in their research paper "Exploring the Gender Divide on YouTube: An Analysis of the Creation and Reception of VLogs," analyzed a random sample of YouTube video blogs or VLogs posted over 15 days, 6 October- 21 October 2016. The content analysis helped the researchers determine major themes of the videos being uploaded, the type of content produced, about the individuals who were VLogging, and the audience's response to each of the VLog. Non-English and longer than three minutes VLogs were omitted from the sample, leaving only 1028 VLogs as the final population. Within this population, every hundredth video was selected for analysis. The VLogs and the VLoggers were coded for different variables, including several views, gender, location, age, audience, technical quality, and messages.

To analyze the gender divide in the audience's responses, half of the participants were males, and another half were female. The research results showed a greater proportion of male VLoggers than female VLoggers, a male to female ratio of 58percent to 33percent (Ibid).

\section{Theoretical Framework}

The medium theory is a relatable approach that discusses the differences in the form of the content and the type of medium used to convey it. It assumes that media, as we consider, is not merely a channel through which the communication occurs. Still, it is a diverse set of environments and settings that enable the communication process, and it can vary in its meaning and sense. Each medium is efficient in its ways, and it can create influence through its standards. (McLuhan, 1962). 
Dr. Ellen Helsper theorizes that it is a basic human instinct to share stories related to their lives. VLogging, according to her, is the next stage of sharing such experiences over the web through online platforms. Users directly participate in the content production process and share the information with others. VLogging, as a medium, relates to this theory as the VLogger records their personal experiences and post them to share with the large audience. However, it lacks real-time face to face communication as it occurs in interpersonal communication. (Helsper, 2015)

Studying the effects of user-generated content through audiovisual media such as YouTube, Corey J Feger hypothesized that medium such as Vlogs provides a new way and avenues to promote resilience and a sense of acceptance otherwise neglected segments of the society. He studied the transgender community to prove that Vlogging is not just a medium of social interaction and sharing information. Still, it can also be used to promote intersectionality in society. Thus, it can bring socio-cultural changes that none of us has anticipated (Feger, 2019).

The rising popularity of Vlogging can be explained through Green, Bobrowicz's work on online videos. He explained that this new trend and a shift towards producing more user-generated content stem from humans' desire to seek support, friendship, and empathy. And to do so, the content producer shares their personal experiences or information with an anonymous audience (Bobrowicz's, 2015)

\section{METHODOLOGY}

This paper's research design is purely quantitative. VLogs from Pakistan are categorized and quantified based on their subscriptions, views, likes, dislikes, comments, the theme, style, and duration of each video blog. This research design was selected based on the previous study named "A content, visual and audience analysis of YouTube VLogs," which closely resembled this research's aim (Molyneux, 2016). Content Analysis can be described as the systematic observation or reading of an artifact or text assigned different labels to discover meaningful facts (Hodder,1994). According to Arthur Asa Berger (2019), The basic assumption implicit in content analysis is that an investigation of messages and communication will allow some insight into some aspect of the people who receive these messages (p. 173).”

A total of 50 VLogs were selected as a sample for this study. The selection was carried out using Purposive Sampling Techniques. A purposive sample is defined as a non-probability sample selected based on the characteristics of a population and the research's set objectives. It is also called selective, judgmental, or even subjective sampling (Crossman, 2018). As W. Lawrence Neuman (2014) pointed out, "we use purposive sampling to identify particular types of cases for an in-depth investigation to gain a deeper understanding of types (p. 274)."

After selecting the number, the videos were picked using the 'most viewed' VLogs from the 'most subscribed' Youtube VLog channels from Pakistan. By setting the views and subscription as a standard bar, the researcher analyzed greater audience interests, inclination, and interactivity. A total of 10 most subscribed Pakistani Youtube VLoggers were selected among all. It is said that the VLoggers, those with 
the highest subscription rates, are more influential, and their effects on viewers are markedly noticed (Burgess and Green, 2009).

The required rating and subscription statistics were sourced from an online platform named 'Social blade'; this site is designed to generate a list of most subscribed from any country, including Pakistan (Kleinberg,2011). All such content producers fitting into the operationally defined characteristics of a VLogger were selected from that list. Further, five VLogs were selected from the 'popular uploads' section of each of the already select VLog channels, and that gave the research a total of fifty videos. Since online subscriptions, views, comments, likes, and dislikes are never static figures, the cutting off time to last record the data was set to be 18:00 on 23rd January 2019.

\section{Research Questions:}

The purpose of this research study is to discover the answers to the following important questions regarding VLogging in Pakistan:

1. Who are the most popular Pakistani video bloggers on Youtube?

2. What are the prominent themes of the sample VLogs?

3. What are the prevalent styles used in the making of the VLogs in Pakistan?

4. What percentage of the audience is actively engaged in the feedback process?

5. Does the duration of the VLogs have an impact on their popularity?

6 . Is there any gender divide or gap among the Vloggers community?

\section{Findings \& Discussion}

Table 1: Most Popular VLoggers from Pakistan:

This table enlists the names and total numbers of subscribers of each VLog channel on Youtube. Based on the subscribers' number calculated as per Your tube's algorithm, the top ten VLoggers from Pakistan were selected.

\begin{tabular}{|l|l|l|l|}
\hline Sr. & VLoggers & Subscribers & Percentages \\
\hline 1 & Irfan Junejo & 547,000 & 31.84 \\
\hline 2 & Taimoor Salahuddin Aka Moroo & 431,000 & 25.09 \\
\hline 3 & The Great Muhammad Ali & 233,191 & 13.58 \\
\hline 4 & Ukhano & 121,913 & 7.10 \\
\hline 5 & Bros Meet the World & 92,000 & 5.36 \\
\hline 6 & Hamza Ibrahim & 88,548 & 5.15 \\
\hline 7 & Momina Munir & 55,972 & 3.26 \\
\hline 8 & Abdullah Khattak & 53,120 & 3.09 \\
\hline
\end{tabular}




\begin{tabular}{|l|l|l|l|}
\hline 9 & The Shehzad Show & 49,909 & 2.38 \\
\hline 10 & Yusraslittlecosmoo & 45,101 & 2.63 \\
\hline & Total & 1717754 & 100 \\
\hline
\end{tabular}

According to this list, Irfan Junejo, with more than 5 million subscribers, tops the list, whereas Taimoor Salahuddin came second with more than 4 million subscribers. As Great Muhammad Ali, the channel stood third in the list with more than 2 million subscribers.

\section{Top 10 Pakistani VLoggers \\ Based on Total no. of subscribers on Youtube Channels}

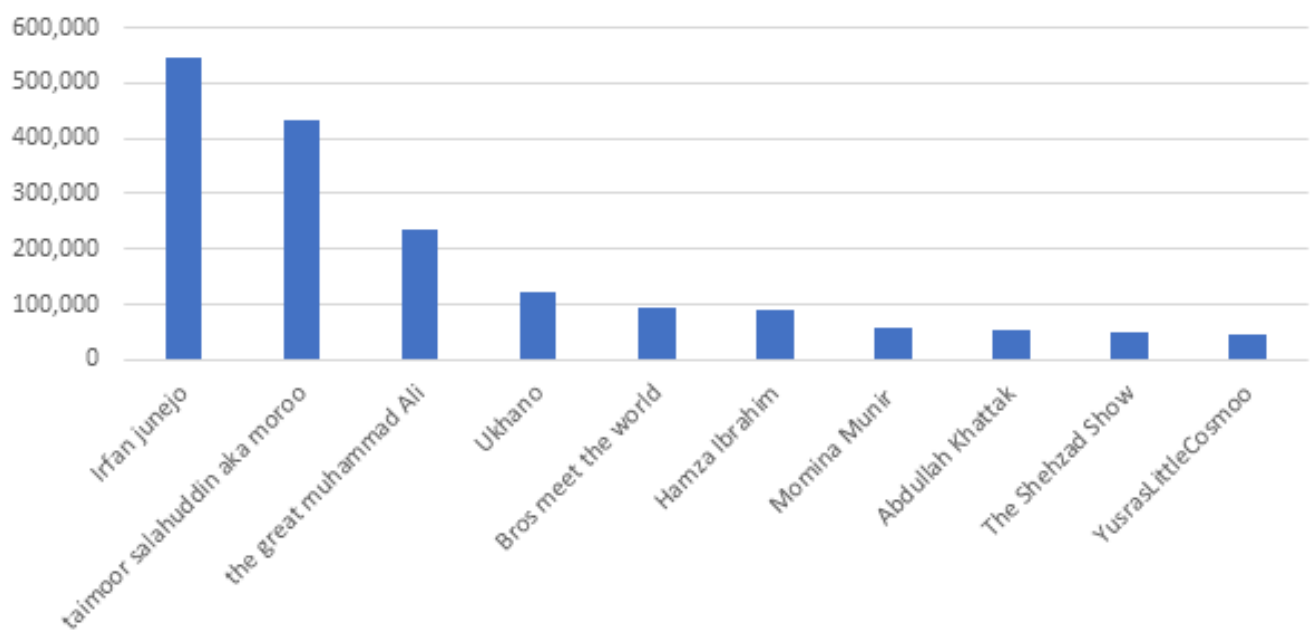

Table 2: VLog Trends

This table enlists all the categories of the themes earlier selected to categorize all the VLogs based on their content. Each video was labeled according to its content with the names of the categories closely related to it.

\begin{tabular}{|l|l|l|l|}
\hline Sr No. & Topics/Themes & Frequency & Percentage \\
\hline 1. & Food & 6 & 8 \\
\hline 2. & Fashion & 2 & 2.67 \\
\hline 3. & Reviews & 1 & 1.33 \\
\hline
\end{tabular}




\begin{tabular}{|l|l|l|l|}
\hline 4. & Technology & 6 & 8 \\
\hline 5. & Games & 0 & 0 \\
\hline 6. & People \& living & 13 & 17.33 \\
\hline 7. & Music/Dances & 3 & 4 \\
\hline 8. & Educational & 6 & 8 \\
\hline 9. & Traveling/Places & 13 & 17.33 \\
\hline 10. & Celebrities/Gossips & 2 & 2.67 \\
\hline 11. & Events/Incidents & 19 & 25.33 \\
\hline 12. & Miscellaneous & 4 & 5.33 \\
\hline & Total & 75 & 100 \\
\hline
\end{tabular}

There were twelve categories selected to identify the trending popular themes among the most viewed VLogs from Pakistan. The category named the "Event/ Incidents" showed the greatest frequency of about 19; its total percentage came out to be 25.33 percent. Two different themes came as second in trend with 17.33 percent: people and living, and the Travelling/Places categories. The category of fashion and celebrities/gossip came third in the trending list, as 2.67 percent of the VLogs contained the content relating to each of the themes.

This result suggests that the VLoggers are most interested in making the eventspecific VLogs for their channels. Whereas they are least interested in producing any content regarding games. They are moderately interested in producing food, technology, and education-related content. Their interest in creating VLogs regarding people and living is almost equal to their interest in traveling related content production.

Table 3: Structure and Style

The style of each of the VLogs was also analyzed and categorized into six distinct categories. This style was determined by the type of gestures, words, and sentence structures used in the VLogs by the content producer. In Style-Shifting in VLogging, a study conducted by Sarah Lee, a similar approach was opted to study the styles. (Lee, 2017)

1. Comedy/Fun/Joyous:

In this type of video style, the VLogger uses humor of all sorts to discuss a certain topic or issue, e.g., puns, jokes, funny gestures, or humorous acts.

2. Formal/Serious:

It is the video style where VLoggers choose a more serious and formal approach to delivering the content to the audience. 
3. Casual Conversational:

In this style, the VLogger breaks the fourth wall and directly addresses the audience in a more interactive style. The content is presented as a one-sided conversation from VLogger to the audience.

4. Tragic/Dramatic:

One enactment, music, and content are used in a VLog to create a tragic or dramatic milieu; such style can be termed as Tragic or dramatic.

5. Satirical:

In most VLogs, the content creators use satirical content to address a certain topic. Such content consists of indirect or direct ridicule, mockery, parody, mimicry, derision, etc.

6. Miscellaneous:

Any other video blogging style not relevant to any of the above categories shall be added to this group.

\begin{tabular}{|l|l|l|l|}
\hline Sr No. & Style/ Structure & Frequency & Percentages \\
\hline 1. & Comedy/Fun/Joyous & 12 & 23.53 \\
\hline 2. & Formal/ Serious & 11 & 21.57 \\
\hline 3. & Casual/Conversational & 19 & 37.25 \\
\hline 4. & Tragic/Dramatic & 3 & 5.88 \\
\hline 5. & Satirical & 3 & 5.88 \\
\hline 6. & Miscellaneous & 2 & 3.92 \\
\hline & Total & 50 & 100 \\
\hline
\end{tabular}

Table no. 3

The most used style in all the VLogs came out to be conversational/casual style with 37.25 percent, whereas 23.53 percent of the VLogs contained comedy/fun style in their content. The least of all the styles used in the VLogs were Dramatic and Satirical, with only 5.88 percent of the VLogs carrying such content.

Table 4: Audience Engagement

While analyzing the popularity and trend of the VLog themes, it was imperative to analyze the audience's engagement in such content. YouTube provides different feedback options to the viewers to allow the video uploader to assess the audience's response accordingly. The total number of views per video on the website includes both the passive audience (the one who only watches the videos) and the active audience (the one which responds to the content of the VLogs). The active audience can either like, dislike, or comment on the video to show their views about the content. By subtracting the total number of likes added to several dislikes and the number of comments from the total number of views, the research was able to find 
the ratio of the active and passive audience engaged with the VLog content.

Total views on the Vlogs $=\mathrm{V}$

The number of views indicates the number of people who have seen or opened the shared vlog on their screens. The total number of views shows the exact "REACH" of a certain Vlog.

$\mathrm{V}=25,723,238$

Active Audience $=\mathrm{A}$

The active audience can be identified through their action of participation or feedback. In the case of YouTube, the engagement can be calculated by adding:

- No. of likes on the videos

- $\quad$ No of dislikes on the videos

- No. of comments on the video

So,

$\mathrm{A}=$ total no. of likes + total no. of dislikes + total no. of comments

- Total no. of like $=586200$

- $\quad$ Total no. of dislikes $=32589$

- $\quad$ Total no of comments $=60653$

$\mathrm{A}=586200+32589+60653=679,462$

The ratio of Active Audience from total Views:

$A=679,462 / 25,723,238 \times 100=2.64 \%$

\section{Passive Audience $=\mathbf{P}$}

By subtracting the calculated audience engagement value from the total number of views, we can calculate the percentage of the audience which remain passive and does not like to interact or give feedback through any given option:

$\mathrm{P}=\mathrm{V}-\mathrm{A}$

$\mathrm{P}=25,723,238-679,462=25,043,776$

The ratio of Passive Audience from total views:

$\mathrm{P}=25,043776 / 25,723,238 \times 100=97.36 \%$

\begin{tabular}{|l|l|l|l|}
\hline & Active Audience & Passive Audience & Total \\
\hline Frequency & 679,462 & $25,043,776$ & 25723238 \\
\hline Percentage & 2.64 & 97.36 & 100 \\
\hline
\end{tabular}

Table no. 4

These percentages indicate that people watching the VLogs are more engaged in viewing them, and only a small fraction of this audience respond and provide their feedback.

Table 5: Video Duration and Popularity

Through the quantitative analysis of all the VLogs, the researchers looked for any relationship between the popularity and the duration of each VLog. To assess the popularity, likes per video were selected as the first variable, and the other variable was the time in minutes per video. The following table enlists all the VLogs with 
their likes and duration in minutes.

\begin{tabular}{|c|c|c|c|c|}
\hline Sr. No & List of VLogs & $\begin{array}{l}\text { Likes Per } \\
\text { Video }\end{array}$ & $\begin{array}{l}\text { Likes } \\
\text { (Percentage) }\end{array}$ & $\begin{array}{l}\text { time } \\
\text { duration } \\
\text { (minutes) }\end{array}$ \\
\hline 1 & $\begin{array}{l}\text { My dream bike- } \\
\text { Yamaha R1 }\end{array}$ & 94,000 & 16.03 & 8.49 \\
\hline 2 & My Yamaha R6 & 37,000 & 6.31 & 6.42 \\
\hline 3 & $\begin{array}{l}\text { the Junejo Guide } \\
\text { to Naran }\end{array}$ & 37,000 & 6.31 & 11.52 \\
\hline 4 & $\begin{array}{l}\text { Lyari underground } \\
\text { live }\end{array}$ & 19,000 & 3.24 & 7.46 \\
\hline 5 & $\begin{array}{l}\text { the coolest way to } \\
\text { go to Hyderabad }\end{array}$ & 34,000 & 5.80 & 6.24 \\
\hline 6 & $\begin{array}{l}\text { Naran with Ahsan } \\
\text { and Junejo }\end{array}$ & 27,000 & 4.61 & 20.4 \\
\hline 7 & $\begin{array}{l}\text { Pakistani Airlines } \\
\text { reviewed }\end{array}$ & 11,000 & 1.88 & 5.46 \\
\hline 8 & $\begin{array}{l}\text { travelling with Mi } \\
\text { part } 1\end{array}$ & 15,000 & 2.56 & 15.48 \\
\hline 9 & $\begin{array}{l}\text { crashed a fortuner } \\
\text { in shigar, jigar } 1 \\
\text { Skardu }\end{array}$ & 13,000 & 2.22 & 12.07 \\
\hline 10 & $\begin{array}{l}\text { VLog- the car } \\
\text { episode }\end{array}$ & 10,000 & 1.71 & 10.47 \\
\hline 11 & $\begin{array}{l}\text { Juhi Chawala } 1 \\
\text { official music } \\
\text { video }\end{array}$ & 43,000 & 7.34 & 3.07 \\
\hline 12 & $\begin{array}{l}\text { Hello friends, gaye } \\
\text { dekh lo }\end{array}$ & 7900 & 1.35 & 8.33 \\
\hline 13 & $\begin{array}{l}\text { People who talk } \\
\text { like this feat } \\
\text { bekaar films }\end{array}$ & 12,000 & 2.05 & 1.47 \\
\hline
\end{tabular}




\begin{tabular}{|c|c|c|c|c|}
\hline 14 & $\begin{array}{l}\text { trippin at Mandi } \\
2017\end{array}$ & 5600 & 0.96 & 4.08 \\
\hline 15 & $\begin{array}{l}\text { Nadir ali dancing } \\
\text { on Juhi Chawala }\end{array}$ & 13,000 & 2.22 & 2.52 \\
\hline 16 & $\begin{array}{l}\text { working with } \\
\text { sonam kapoor? }\end{array}$ & 16,000 & 2.73 & 6.27 \\
\hline 17 & $\begin{array}{l}\text { skardu "heaven on } \\
\text { earth." }\end{array}$ & 12,000 & 2.05 & 18.57 \\
\hline 18 & $\begin{array}{l}\text { First time in Hunza } \\
\text { with MI }\end{array}$ & 6600 & 1.13 & 15.19 \\
\hline 19 & $\begin{array}{l}\text { First time in } \\
\text { Karachi }\end{array}$ & 4600 & 0.78 & 25.48 \\
\hline 20 & LUMS & 3800 & 0.65 & 8.01 \\
\hline 21 & $\begin{array}{l}\text { Hussainabad ka } \\
\text { protein shake }\end{array}$ & 4700 & 0.80 & 6.06 \\
\hline 22 & Karachi street food & 7600 & 1.30 & 9.39 \\
\hline 23 & VLog 036 & 5100 & 0.87 & 10.37 \\
\hline 24 & $\begin{array}{l}\text { the craziest food } \\
\text { street in Karachi }\end{array}$ & 6600 & 1.13 & 11.42 \\
\hline 25 & VLog 031 & 4800 & 0.82 & 13.04 \\
\hline 26 & $\begin{array}{l}\text { A local boy of } \\
\text { Turkey }\end{array}$ & 16,000 & 2.73 & 13.04 \\
\hline 27 & $\begin{array}{l}\text { VLog } 41 \text { my new } \\
\text { bike }\end{array}$ & 14,000 & 2.39 & 9.06 \\
\hline 28 & $\begin{array}{l}\text { my second VLog } 1 \\
\text { Bollywood boy in } \\
\text { UAE }\end{array}$ & 11,000 & 1.88 & 10.53 \\
\hline 29 & $\begin{array}{l}\text { my first VLog- } \\
\text { Lassan Lag Gaye }\end{array}$ & 12,000 & 2.05 & 4.24 \\
\hline
\end{tabular}


ILMA Journal of Social Sciences \& Economics (IJSSE) Volume 1 Issue 1 January 2021

\begin{tabular}{|c|c|c|c|c|}
\hline 30 & $\begin{array}{l}\text { how to VLog with } \\
\text { a phone }\end{array}$ & 14,000 & 2.39 & 5.32 \\
\hline 31 & $\begin{array}{l}\text { school life in } \\
\text { Pakistan }\end{array}$ & 4500 & 0.77 & 5.46 \\
\hline 32 & $\begin{array}{l}\text { Farewell day } 1 \text { Last } \\
\text { year of school ends }\end{array}$ & 3400 & 0.58 & 14.51 \\
\hline 33 & $\begin{array}{l}\text { My Eid dress was } \\
\text { hella annoying }\end{array}$ & 3700 & 0.63 & 7.27 \\
\hline 34 & $\begin{array}{l}\text { No, Bro, I don’t } \\
\text { Copy Irfan Junejo }\end{array}$ & 2100 & 0.36 & 7.06 \\
\hline 35 & $\begin{array}{l}\text { Never let your } \\
\text { friend's VLog for } \\
\text { you }\end{array}$ & 3400 & 0.58 & 7.13 \\
\hline 36 & $\begin{array}{l}\text { a day at beacon- } \\
\text { house Peshawar }\end{array}$ & 5600 & 0.96 & 8.47 \\
\hline 37 & $\begin{array}{l}\text { Are you single? } \\
\text { Q/A }\end{array}$ & 4500 & 0.77 & 9.39 \\
\hline 38 & Hamara Farewell & 2700 & 0.46 & 4.27 \\
\hline 39 & $\begin{array}{l}\text { Live reaction to } \\
\text { my AS Level CIE } \\
\text { result }\end{array}$ & 4400 & 0.75 & 5.51 \\
\hline 40 & Pakistan mei Eid & 3,000 & 0.51 & 4.37 \\
\hline 41 & haan Bhaiya & 4100 & 0.70 & 6.14 \\
\hline 42 & I copy Irfan junejo & 3100 & 0.53 & 11.03 \\
\hline 43 & Mission Shaadi & 2400 & 0.41 & 7.49 \\
\hline 44 & Mission Eid & 3500 & 0.60 & 12.34 \\
\hline 45 & Zero to a Hundred & 5100 & 0.87 & 7.46 \\
\hline 46 & $\begin{array}{l}\text { FROM } \\
\text { AUSTRALIA TO } \\
\text { PAKISTAN. }\end{array}$ & 2700 & 0.46 & 4.44 \\
\hline
\end{tabular}




\begin{tabular}{|c|l|l|l|l|}
\hline 47 & $\begin{array}{l}\text { BROWN PEOPLE } \\
\text { VALIMA VLOG }\end{array}$ & 1700 & 0.29 & 3.03 \\
\hline 48 & $\begin{array}{l}\text { WELCOMING } \\
\text { OUR BRIDE }\end{array}$ & 8700 & 1.48 & 7.15 \\
\hline 49 & $\begin{array}{l}\text { PAKISTANI } \\
\text { BROWN } \\
\text { MEHANDI VLOG }\end{array}$ & 140 & 0.02 & 20.15 \\
\hline 50 & $\begin{array}{l}\text { WELCOMING } \\
\text { MY NEPHEW }\end{array}$ & 180 & 0.03 & 9.54 \\
\hline & Total & 586,220 & 100 & 451.68 \\
\hline
\end{tabular}

Table no. 5

Following is the graphical representation of popularity against their durations. The total variables for each VLog are represented using line and bar graphs. The bars represent several likes, and the peaks of the line graph show the duration per VLog. By comparing the peaks with the bars' height, the researchers analyzed the relation between the two variables. According to this graph chart, the lower the duration peaks, the higher the popularity bar is, and that means that the duration has an inverse relation to the likeness of the VLogs by the audience. The longer the VLog, the lesser the audience will click the like button for each VLog.

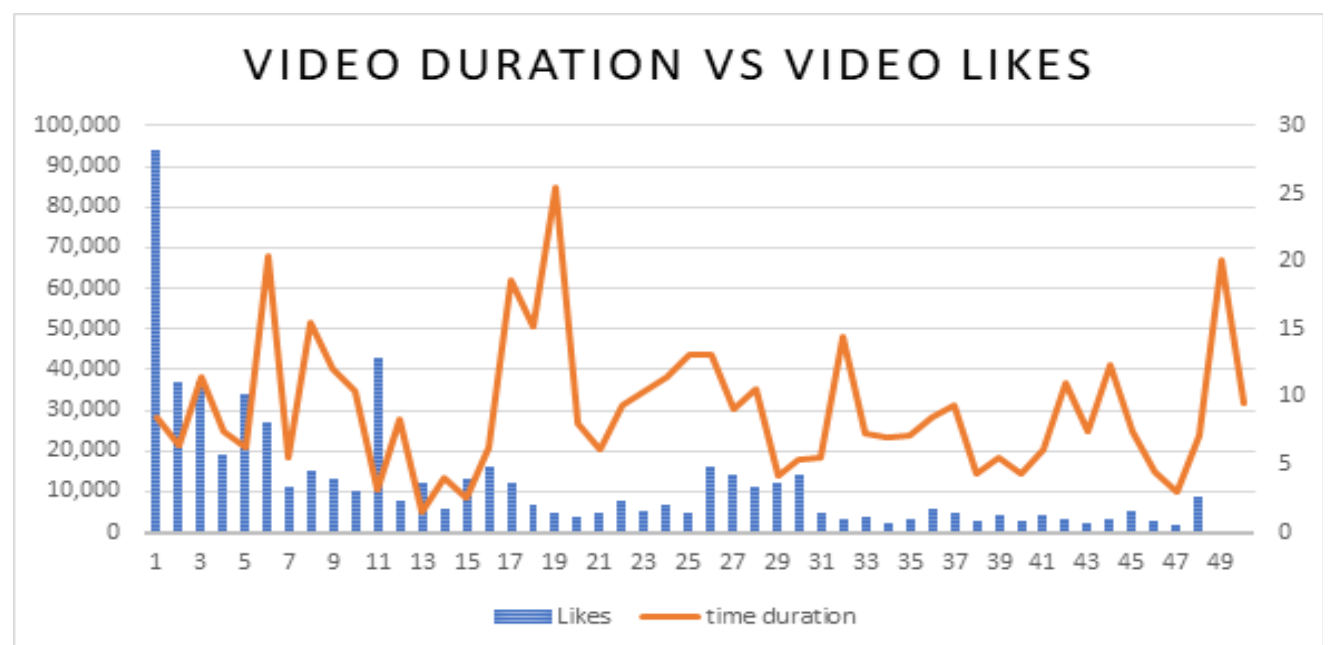

\section{Table 6: Male vs. Female VLoggers}

VLogs were also subjected to analyze the gender disparity among the content producers. That can be done by calculating the percentages of the male and the female VLoggers among all the popular VLoggers from Pakistan. 


\begin{tabular}{|l|l|l|l|l|}
\hline & & Male & Female & Total \\
\hline \multirow{2}{*}{$\begin{array}{l}\text { Total no. of } \\
\text { channels }\end{array}$} & $\mathrm{F}$ & 8 & 2 & 10 \\
\cline { 2 - 5 } & $\mathrm{P}$ & 80 & 20 & 100 \\
\hline \multirow{2}{*}{ Total VLogs } & $\mathrm{F}$ & 40 & 10 & 50 \\
\cline { 2 - 5 } & $\mathrm{P}$ & 80 & 20 & 100 \\
\hline \multirow{2}{*}{ Total views } & $\mathrm{F}$ & $22,941,331$ & 2781907 & 25723228 \\
\cline { 2 - 5 } & $\mathrm{P}$ & 89.19 & 10.81 & 100 \\
\hline
\end{tabular}

Table no. 6a

Among the top ten VLog channels in Pakistan, eight channels are run by male VLoggers, whereas only two of them are female VLoggers. That translates into an $80 / 20$ male to female VLogger ratio. A similar difference was obtained when the total number of VLogs was calculated against male and female categories, which turned out to be 80 and 20 percentages, respectively, which means that more videos were created by the male content producers than the female producers. The total views for VLog produced by males were also far greater in proportion than for the VLogs made by the females, with 89.19 percent for males and 10.81 percent for females. All these statistics indicate that gender disparity exists in the popularity of the VLoggers and their VLogs. From Pakistan, more male VLoggers enjoy the most subscribers and views on their VLogs than the female VLoggers, which indirectly impacts the trending themes of VLogs uploaded for the audience.

\begin{tabular}{|l|l|l|l|l|}
\hline & Male & Females & Total & \\
\hline \multirow{2}{*}{ Subscriptions } & $\mathrm{F}$ & 1616681 & 101073 & 1717754 \\
\cline { 2 - 5 } & $\mathrm{P}$ & 94.12 & 5.88 & 100 \\
\hline \multirow{2}{*}{ Likes } & $\mathrm{F}$ & 555700 & 30520 & 586220 \\
\cline { 2 - 5 } & $\mathrm{P}$ & 94.7 & 5.2 & 99.9 \\
\hline \multirow{2}{*}{ Dislikes } & $\mathrm{F}$ & 27625 & 4964 & 32589 \\
\cline { 2 - 5 } & $\mathrm{P}$ & 84.7 & 15.2 & 99.9 \\
\hline
\end{tabular}

Table no.6b

Table $6 \mathrm{~b}$ shows enough difference between the total subscriptions gained by the male VLoggers and Female VLoggers. Male VLoggers showed about 94.12 percent of the 10 samples YouTube channels' total subscriptions, whereas the Female VLogger has 5.88 percent of the total subscriptions. Similarly, the difference exists 
between the total likes on all the videos by male and female VLoggers, 94.7percent for males and 5.2 percent for the female's VLoggers. The dislikes showed a slightly different trend, with male VLoggers receiving 84.7 percent of the total dislikes on all the videos combined. Female VLoggers 15.2 percent of the total dislikes on all the videos collectively.

\section{CONCLUSION:}

This research study over the contemporary trends and popularity of the VLogs as the emerging new medium in Pakistan was able to find out many answers regarding the entire phenomenon of VLogging in the country.

RQ1: Who are the most popular Pakistani video bloggers on YouTube?

The number of subscribers helped the researcher to set the direction straight. These figures helped identify the top trending Vloggers in Pakistan in the given period. With the highest number of subscribers, five million, and forty-seven thousand, the Vlogger is marked as the most popular and trending Vlog celebrity in the country.

RQ2: What are the prominent themes of the sample VLogs?

The trends and themes of the VLogs were determined. The result from that content analysis suggested that people on YouTube are more interested in sharing information regarding events or incidents through this medium. As per Helsper's prescription, people like to share more about the experiences that they likely experience (Helsper, 2015). VLogs regarding the traveling places and people are the next major theme the VLoggers likely to select for their VLogs. There is far less inclination towards gaming, food, fashion, and technology in the content of popular VLogs from Pakistan. That indicates the general interest of the audience in different themes.

RQ3: What are the prevalent styles used in the making of the VLogs in Pakistan? When it comes to the style and structure of the VLogs, most of the content producers used a casual/conversational style while addressing the audience. In the light of McLuhan's medium theory, each medium uses a distinct feature for successful dissemination of content; in the case of the selected sample VLogs, the casual style and structure of the content seem more suitable (McLuhan, 1962). They were least likely to select a satirical or dramatic style of addressing the audience or delivering the message. The use of humor is also popular after the casual style, followed by the formal style. The analysis of the styles enables us to conclude that the casual or conversational style of sharing information through VLogs is significant in creating the impact.

RQ4: What percentage of the audience is actively engaged in the feedback process? The results showed that this medium, despite enjoying viewership by millions and thousands of people, fails to engage the audience in the feedback process, either direct or indirect. There was a minor proportion of 3 percent approximately, which provides the feedback, whereas 97 percent still does not provide their feedback. This gap between active and passive audiences shows that VLogging in Pakistan lacks the basic elements of direct feedback, which essentially controls the direction 
of the content produced.

RQ5: Does the duration of the VLogs have any impact on their popularity or likeness among the audience?

The relation of video blog duration to its popularity was also determined to understand whether this variable impacts the likeness of the content by the audience; it turned out that the longer the VLogs, the lesser the audience pressed the like button for them. That suggests that VLoggers should consider making short and comprehensive video blogs to positively engage their audience.

RQ6: Is there any gender divide in the Vloggers community?

The gender disparity in the medium of analysis was studied by comparing the number of the channels run by the male and female VLoggers; and the number of VLogs created by them. The figures showed that more male VLoggers found their place in the top ten VLoggers in Pakistan, and only a small percentage of females became part of this list. Hence the themes and topics covered in the popular VLogs are more male-centric than females. Consequently, those VLogs largely serve the needs and interests of the male audience than the female ones.

It is believed that in patriarchal societies like Pakistan, female content producers are rare to find. However, the results from this research not only showed that the females are successfully running their vlog channels, but they also made it to the list of top ten most popular Vloggers in Pakistan. And these findings suggest what Feger (n.d.) had pointed out that vlogs do prove to be a medium of convenience for those who do not generally get to share their views and thoughts with society. Hence, as long as vlogging is concerned, women from the country can independently use vlogs as their medium of expression.

In a nutshell, a preliminary study, as this one, can only provide the basis to detect the emergence of the use, trends, and impact of a medium like VLogs. The established links between the themes, their use, popularity, audience engagement, VLog's style, structure, and gender disparity can prove to be foundational when conducting an in-depth analysis of a single aspect of VLogging in Pakistan. A country, which has been facing a technology lag in today's multifaceted cyber world, should look towards VLogging with a more serious approach and a clearer direction.

\section{REFERENCES}

Aran, O., Biel, J., \& Gatica-Perez, D. (2014). Broadcasting Oneself: Visual Discovery of Vlogging Styles. IEEE Transactions on Multimedia, 16(1), pp. 201-215. doi:10.1109/tmm.2013.2284893

Bal, M. (n.d.). Visual Analysis. The SAGE Handbook of Cultural Analysis, pp. 163-184. doi:10.4135/9781848608443.n9

Biel, J., \& Gatica-Perez, D. (2013). The YouTube Lens: Crowdsourced Personality Impressions and Audiovisual Analysis of Vlogs. IEEE Transactions on Multimedia, 15(1), pp. 41-55. doi:10.1109/tmm.2012.2225032

Berger, Arthur Asa (2019). Media Analysis Techniques, edn 6, Sage Publications, Inc., USA, p .173. 
Cha, M., Kwak, H., Rodriguez, P., Ahn, Y., \& Moon, S. (2007). I tube, you tube, everybody tubes. Proceedings of the 7th ACM SIGCOMM Conference on Internet Measurement - IMC '07. doi:10.1145/1298306.1298309

Chong, C., Molyneaux, H., \& Fournier, H. (2012). Communication as Commodification. The Handbook of Gender, Sex, and Media, pp. 419-435. doi:10.1002/9781118114254.ch25

Croteau, D. (2006). The Growth of Self-Produced Media Content and the Challenge to Media Studies. Critical Studies in Media Communication, 23(4), pp. 340344. doi:10.1080/07393180600933170

Feger, C. (n.d.). Vlogging truth to power: A qualitative study of resilience as practiced by transgender youtube content creators. doi:10.18297/etd/3185

Green, M., Bobrowicz's, A., \& Ang, C. S. (2015). The lesbian, gay, bisexual and transgender community online: Discussions of bullying and self-disclosure in YouTube videos. Behavior \& sInformation Technology, 34(7), pp.704-712. do i: $10.1080 / 0144929 x .2015 .1012649$

Griffith, M., \& Papacharissi, Z. (2009). Looking for you: An analysis of video blogs. First Monday. doi:10.5210/fm.v15i1.2769s

Hansen, A., Cottle, S., Negrine, R., \& Newbold, C. (1998). Content Analysis. Mass Communication Research Methods, pp. 91-129. doi:10.1007/978-1-34926485-8 5

Lee, S. (2017). Style-Shifting in Vlogging: An Acoustic Analysis of "YouTube Voice". Lifespans and Styles, 3(1), 28-39. doi:10.2218/ls.v3i1.2017.1826

Lee, S. (2017). Style-Shifting in Vlogging: An Acoustic Analysis of "YouTube Voice". Lifespans and Styles, 3(1), pp. 28-39. doi:10.2218/ls.v3i1.2017.1826

Neuman, W. Lawrence (2014). Social Research Methods: Qualitative and Quantitative Approaches, edn, 7th, Pearson Education 1td, USA.

Pedersen, S., \& Macafee, C. (2007). Gender Differences in British Blogging. Journal of Computer-Mediated Communication, 12(4), pp. 1472-1492. doi:10.1111/ j.1083-6101.2007.00382.x

Rethinking Media Change. (2003). doi:10.7551/mitpress/5930.001.0001

Rust, J., \& Altman, S. (2020). Vlogging as Sense Making. Fieldnotes in Qualitative Education and Social Science Research, pp. 217-233. doi:10.4324/9780429275821-18

Snelson, C. (2013). Vlogging about school on YouTube: An exploratory study. New Media \& Society, 17(3), pp. 321-339. doi:10.1177/1461444813504271

Vlogging. (2014). Encyclopedia of Social Media and Politics. doi:10.4135/9781452244723.n564

YouTube: Online video and participatory culture. (2010). Choice Reviews Online, 47(06). doi:10.5860/choice.47-2989 